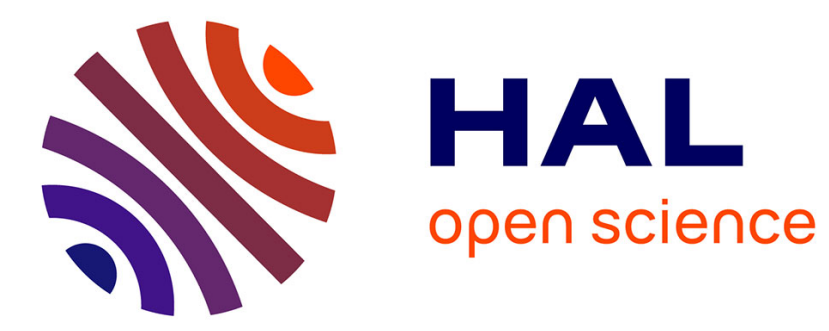

\title{
From reverse engineering to shape engineering in mechanical design
}

\author{
Nabil Anwer, Luc Mathieu
}

\section{To cite this version:}

Nabil Anwer, Luc Mathieu. From reverse engineering to shape engineering in mechanical design. CIRP Annals - Manufacturing Technology, 2016, 10.1016/j.cirp.2016.04.052 . hal-01363747

\section{HAL Id: hal-01363747 \\ https://hal.science/hal-01363747}

Submitted on 11 Sep 2016

HAL is a multi-disciplinary open access archive for the deposit and dissemination of scientific research documents, whether they are published or not. The documents may come from teaching and research institutions in France or abroad, or from public or private research centers.
L'archive ouverte pluridisciplinaire HAL, est destinée au dépôt et à la diffusion de documents scientifiques de niveau recherche, publiés ou non, émanant des établissements d'enseignement et de recherche français ou étrangers, des laboratoires publics ou privés. 


\title{
From Reverse Engineering to Shape Engineering in Mechanical Design
}

\author{
Nabil Anwera, Luc Mathieua
}

a LURPA, ENS Cachan, Univ. Paris-Sud, Université Paris-Saclay, 94235 Cachan, France

Reverse engineering and shape reconstruction play an important role in design and manufacturing through the increased use of shape acquisition and processing technologies in the product development process. The application of shape theories to geometric modelling and variability characterization are paving the way to shape engineering and more generic methods for reverse engineering.

This paper investigates the fundamentals of shape representation, shape processing and mining at a conceptual, geometric and computational level to address geometric reverse engineering issues in mechanical design. New developed concepts based on discrete curvatures and their applications are presented. Challenges and future researches are also highlighted.

Reverse Engineering, Geometric Modelling, Product Development

\section{Introduction}

Nowadays, reverse engineering is recognized as an important issue in the product design process which highlights inverse methods, deduction and discovery in design. In mechanical engineering, reverse engineering has evolved from capturing technical product data, and initiating manual redesign procedure while enabling efficient concurrency benchmarking to a more elaborated process based on advanced computational models and modern digitizing technologies.

The reverse engineering of mechanical products is generally addressed in both the practice and the literature from a geometric or shape perspective. Geometric reverse engineering relies on a set of generic methods inherited from the geometric modelling and processing fields. Those methods encompass mesh segmentation, surface reconstruction, and feature recognition. Moreover, geometric reverse engineering is nowadays supported by a digital thread from the raw acquired point cloud data to parametric feature-based CAD models. In this context, it envisions a more global geometric reverse engineering process with a life cycle perspective (figure 1).

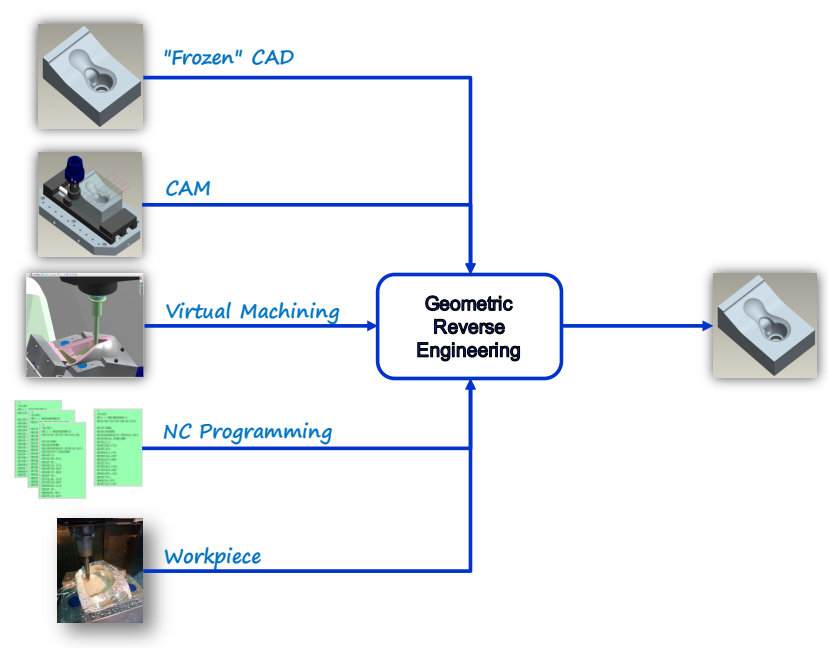

Figure 1. The global context of geometric reverse engineering.
It enables also an improved reverse engineering process through knowledge-based techniques to make more explicit and sharable the knowledge embedded in the digital flow, and recently, it draws the attention to the convergence of reverse engineering, metrology, and software for additive manufacturing-related applications [1].

Lying at the interface of mechanical engineering, modern geometry, computer science, and statistics, shape engineering is concerned with the study of geometric properties of mechanical parts and assemblies. Its goal is to establish a theoretical foundation for the modelling and processing of product's shape and variability, and to develop methods for geometric reasoning to infer knowledge and enable decision making in the product development process [2].

This paper investigates the fundamentals of shape representation, shape processing and mining at a conceptual, geometric and computational level to address geometric reverse engineering issues in mechanical design. New developed concepts based on discrete curvatures and their applications are presented. Challenges and future researches are also highlighted.

\section{Reverse engineering vs. geometric reverse engineering}

Although reverse engineering theories and methods, traced back to the 1980s, were predominant in the areas of software, hardware and biological systems, reverse engineering techniques are applied in different areas [3-6], ranging from mechanical engineering, civil engineering, architecture, cultural heritage, to dentistry and medicine.

\subsection{Reverse engineering}

Reverse engineering was originally defined as "the process of developing a set of specifications for a complex hardware system by an orderly examination of specimens of that system" [7]. Chikofsky and Cross [8] defined reverse engineering as "the process of analysing a subject system to identify the systems components and their relationships, and to create representations of the system in another form or at a higher level of abstraction". 
Reverse engineering is generally addressed in the literature from different perspectives that include system benchmarking and recovery, and inverse problem modelling to infer a model and its parameters from experimental data [9].

In mechanical design, reverse engineering can be defined as the process that "initiates the redesign process wherein a product is predicted, observed, disassembled, analyzed, tested, 'experienced', and documented in terms of its functionality, form, physical principles, manufacturability, and assemblability" [10]. It has been considered a method to understand how a product works [11], and the process of duplicating an object to obtain a surrogate model or a clone to enhance its performance, and to capture and apply the embedded knowledge to new design.

\subsection{Geometric reverse engineering}

The consideration of the geometrical aspect of the product has led to a tremendous growth in research on what is called, variously, geometric reverse engineering or reverse geometric modelling [12].

The extraction of geometry from an existing product to reconstruct a 3D CAD model is the most used approach for geometric reverse engineering. Though there exist multiple descriptions of the geometric reverse engineering process, they can all be condensed to three main steps: product digitization, shape reconstruction, and 3D CAD modelling. This process can be iterative in nature (figure 2).

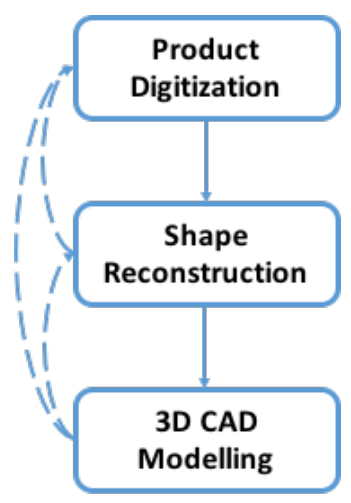

Figure 2. Basic phases of geometric reverse engineering.

Product digitization refers in general to the phase of digitizing a physical product using measurement and scanning devices. It can be extended to include all other processes that can define a virtual product or its signature such as mechanical simulation, manufacturing process simulation and shape and topology optimization. Thus, geometric reverse engineering focuses not only on the reconstruction of the shape from measurement, but also on the integration of materials properties and manufacturing processes and their inherent variability.

Geometric modelling and computational geometry play an important role in the geometric reverse engineering process. Indeed, they cover a geometric and digital thread that include physical (real), mathematical (abstract), and discrete (digital) representations of shapes and the transition mechanisms between them [13]. The underlying mathematical developments focus on developing discrete and computational geometry methods acting on point clouds and enabling dedicated algorithms to address completeness, uncertainty, and sparsity of data.

The step of shape reconstruction is to determine a surface that approximates an unknown shape from samples. This problem is illdefined since many surfaces approximating the samples can be retrieved. Moreover, the point set can be characterized with variable density as well as noise and outliers due to the acquisition process. The main challenge here is to guarantee that the topology of the original surface is preserved, while sharp features and surface boundaries are reproduced accurately in the reconstructed surface. The research literature is very rich and various techniques have been developed. The earliest techniques used NURBS or BSplines to fit and stitch together local surface patches but showed their limits when dealing with complex physical objects, large data, sparsity and noise. Moreover, they are not applicable when physical properties like material or density of the object need to be considered.

3D CAD modelling is the final step to create a geometric (solid) product model using a Boundary Representation (B-Rep) or a feature-based parametric representation to embed design intents through geometric features, parameters and constraints

Feature-based parametric models are often required for model query and interrogation or design modification. Moreover, they enable the development of Knowledge-Based Reverse Engineering (KBRE) methodologies to include more a-priori information to overcome noisy or missing data and to extract more design and manufacturing data [14-15].

\section{Shape framework for geometric reverse engineering}

The study of shape has a long and rich history. It has been highlighted in different areas such as philosophy, psychology, mathematics, and engineering for understanding the mechanisms of perception, to emulate the human visual system, and to address geometric and structural data of natural and man-made objects.

\subsection{Shape Conceptualization}

According to the Merriam-Webster Dictionary a shape is "the visible makeup characteristic of a particular item or kind of item," "a spatial form or contour," or "a standard or universally recognized spatial form." Thus, the geometrical properties of shape have generated the most interest from the researchers since the pioneering work of D'Arcy Thompson and Kendall [16]. An intuitive definition of shape given by Kendall is: "shape is all the geometrical information that remains when location, scale, and rotational effects are filtered out from an object." Hence, this definition allows for a similarity measure among shapes, which is the fundamental of Statistical Shape Analysis (SSA) [17]. Moreover, SSA methods establish, from a training set, the pattern and the parameters of variation in the shapes and their spatial relationships. Thus, an efficient parameterization of this variability can be established which provides a compact representation of shapes. SSA methods have been successfully applied to Geometrical Product Specifications (GPS) thanks to Skin Model Shapes paradigm [18].

\subsection{Shape representation and description}


A shape model is a computational structure that captures the spatial aspects of the product. Shape representation can be defined as a mapping from a computer structure to a well-defined mathematical model which defines the real or physical product in terms of computable mathematical properties [19]. These properties for shape representation include shape type (discrete or continuous), structural expression (point-based, surface/shellbased or volume-based), and the representation scheme (enumerative, cell complex, functional or constructive).

While shape representation contains enough information to reconstruct the product, shape description more likely contains enough information to identify, analyse, understand, and recognize shapes [20]. Through shape descriptors, a more compact and concise representation is enabled. Various descriptors have been reported in the literature such as shape signature, Fourier descriptors, medial axis, curvature, etc. [21].

Since a cloud of points does not infer too much information about the digitized product, a polyhedral or triangular mesh is constructed in order to organize the points and reconstruct the topology of the product. Curvature is a shape descriptor and a shape invariant parameter. It is one of the most important differential character of the shape. Reliable estimation of discrete curvatures is often required in many applications when the surface is defined by a set of discrete points.

\section{Shape processing for geometric reverse engineering}

In this paper, we use a discrete shape representation, a curvature-based description and a shape clustering approach to illustrate the above-mentioned shape related concepts in the context of geometric reverse engineering.

Segmentation is one of the most critical issues in shape processing for geometric reverse engineering. It is used to decompose the digitized product into a set of meaningful regions. Hence, our proposed method is based on shape segmentation [22] and comprises four steps (figure 3): discrete curvature estimation, boundary identification, vertex clustering, and connected region generation.

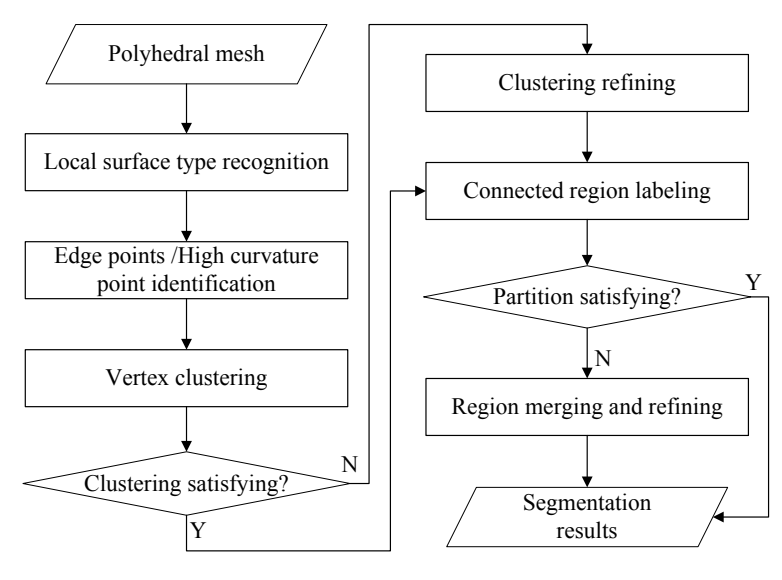

Figure 3. Shape segmentation process.

\subsection{Discrete curvature estimation}

The theory of the perception of shape of Koenderink and Doorn [23] suggest a new set of curvature-based shape descriptors. The Shape Index (s) is a measure of the shape, modulo its size. It defines a bounded and continuous measure on the interval $[-1 ; 1]$. It is determined by the following formula (1):

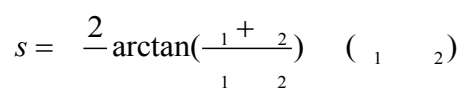

Where $\kappa_{1}$ and $\kappa_{2}$ are the maximum and minimum principal curvatures at the considered point.

This definition does not apply to the special case of the planar shape because the two principal curvatures are null. Hence, we have extended the definition of Koenderink to flat surfaces by assigning the value of 2 to this element.

A Shape Index color map was also introduced by Koenderink [23]. A color scale is associated to each Shape Index interval (figure 4), and a black color is added to highlight planar shapes.

Another curvature parameter was introduced by Koenderink to characterize more intuitively the curvature intensity than the Mean and the Gaussian curvatures. The Curvedness (c) or Casorati curvature is a characteristic of the size that is given by the following formula (2):

$$
c=\sqrt{\frac{{ }^{2}+{ }_{2}^{2}}{2}}
$$

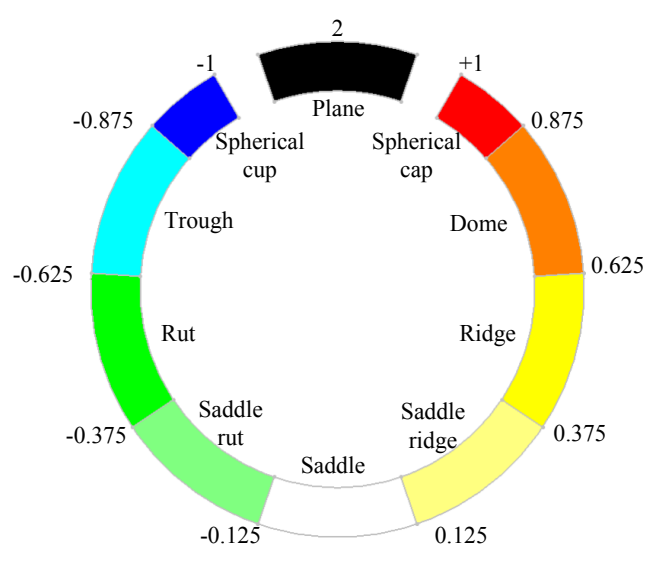

Figure 4. Shape index color map.

The pair of shape descriptors $(\mathrm{s}, \mathrm{c})$ are the basis of our work on discrete shape processing and segmentation [22].

\subsection{Boundary identification}

Here, we propose a curvedness based method to identify edges and boundaries. Figure 5 gives examples of curvedness map for two mechanical parts. From the curvedness map, we can find that the boundary points usually have the larger curvedness values which are distinct from the points in other regions. The boundary points can then be identified as the points which have the curvedness value larger than a given threshold. 

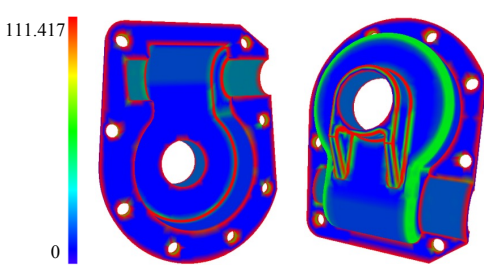

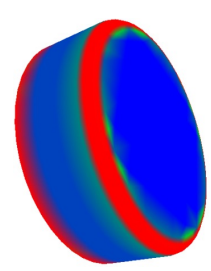

Figure 5. Curvedness map.

\subsection{Vertex clustering}

Vertices are first clustered into 10 different clusters according to their local surface types (figure 4). However, due to sparsity and noise of the acquired point data and the computational errors, the initial clustering results are often imperfect and need to be refined (figure 6).
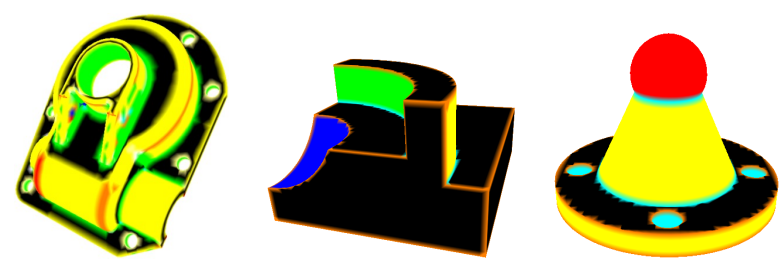

Figure 6. Examples of initial vertex clustering.

The cluster refining algorithm is based on an iterative process. At each iteration, given a vertex, we firstly record all the surface types in its vicinity region. Secondly, we calculate the possibility of every appeared surface type that the given vertex should be refined to. Finally, the surface type of the given vertex is refined to the cluster whose surface type has the maximum possibility. The iteration terminates when the surface type of each vertex doesn't change or a user-defined convergence condition is satisfied.

In cluster refining, we use cluster distance to measure the similarity between two clusters. The cluster distance measures the similarity of local surface types between two vertices. The value range of cluster distance is $[0 ; 1]$. The smaller cluster distance implies that the similarity between the surface types of the two vertices is larger, and the two vertices have a highest possibility to be refined within the same surface type.

\subsection{Connected region generation}

In general, every cluster contains several connected regions. The main purpose of connected region generation is to identify these connected regions and generate the segmentation result of a given discrete model.

The proposed method contains two basic steps: (a) connected region labelling, which is performed to generate the initial segmentation result; (b) region refining, which is used to merge the small regions and improve the quality of the segmentation result.

\subsection{Feature recognition}

A feature is a distinctive object in a product with geometric and topological characteristics. It can be associated with a function or manufacturing operation. Most common feature recognition methods consist in searching a solid's boundary for pattern of faces and edges that obey certain topological or geometrical relationships. Feature recognition methods addressed mainly the machining domain for process planning and integration of CAD and CAM systems.

In the above context of shape modelling, a feature is defined as a set of connected regions satisfying certain geometrical and topological conditions. After the regions are identified, adjacency between regions is constructed, which is then used to recognize features (figure 7). A rule based manufacturability analysis approach is used in our work for feature recognition in virtual machining [24]. The rules are designed as per STEP-NC feature definitions (ISO 14649) so that the recognized feature data can be stored in conformance to STEP-NC. The rule-based feature recognition methodology based on topological combinations of regions is quite general and adaptable and can be easily extended to additive manufacturing which represent other emerging areas of great potential importance for feature recognition.

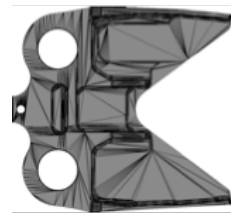

(a) NC Simulation

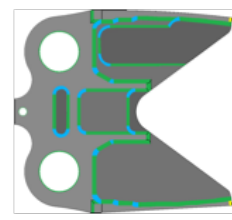

(b) Segmentation

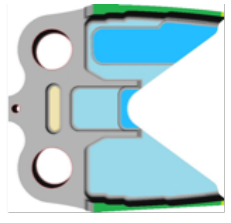

(c) Feature Recognition
Figure 7. Feature recognition from NC simulation.

\subsection{Discussions}

The above proposed methods for shape segmentation and feature recognition have been successfully tested on both engineering products and freeform shapes. The limitations of these methods were considered and compared with other approaches. Foremost among these limitations are: (i) nonsmoothness of the boundaries of the shapes in the case of complex objects, (ii) sampling from rough surfaces, (iii) noise and sparsity of data, (iv) processing large flow of data, and (v) lack of external source of information and knowledge.

\section{Conclusion}

In this paper, we showed that many insights of reverse engineering in the product development process may be gained from novel concepts, methods and tools of shape engineering. Geometric reverse engineering has been extended to capture the shape essence of the physical product, manufacturing processes as well as digital product within a lifecycle perspectice. Shape engineering enables not only a comprehensive framework for shape representation but also a set of methods and tools for shape processing, analysis and mining. 
Shape segmentation and shape/feature recognition are foundametal probems in shape processing for geometric reverse engineering. Through the discrete curvatures, a novel framework has been proposed and successfully tested on engineering products.

Future work will involve enhancing our clustering method to address shape mining and explore other potential applications from spatial data mining domain. Additive manufacturing is another area of future research. Reverse engineering topology optimization outputs in the context of additive manufacturing topology will be considered in the future.

\section{References}

[1] Macy B, 2015, Reverse Engineering for Additive Manufacturing. Handbook of Manufacturing Engineering and Technology, Springer, London 2485-2504.

[2] Schleich B, Anwer N, Mathieu L, Wartzack S, 2014, Skin Model Shapes: A new paradigmshift for geometric variations modelling in mechanical engineering. Computer-Aided Design 50:1-15.

[3] Stark R, Grosser H, Müller P, 2013, Product analysis automation for digital MRO based on intelligent 3D data acquisition. CIRP Annals-Manufacturing Technology 62(1):123-126

[4] Majstorovic V, Trajanovic M, Vitkovic N, Stojkovic M, 2013, Reverse engineering of human bones by using method of anatomical features. CIRP AnnalsManufacturing Technology 62(1):167-170.

[5] Erdős G, Nakano T, Váncza J, 2014, Adapting CAD models of complex engineering objects to measured point cloud data. CIRP Annals-Manufacturing Technology 63(1):157-160.

[6] Laroche F, Bernard A, Hervy B, 2015, DHRM: A new model for PLM dedicated to product design heritage. CIRP Annals-Manufacturing Technology 64(1):161164.

[7] Rekoff M, 1985, On reverse engineering. IEEE Transactions on Systems, Man and Cybernetics 3/4:244-252.

[8] Chikofsky E, Cross J, 1990, Reverse engineering and design recovery: A taxonomy. IEEE Software 7(1):13-17.

[9] Kirk P, Silk D, Stumpf M P, 2016, Reverse engineering under uncertainty, Uncertainty in Biology. Springer International Publishing 15-32.

[10] Otto K, Wood K, 1998, Product Evolution: A reverse Engineering and Redesign Methodology, Research in Product Development 10(4):226-243.

[11] Ullman D G, 2010, The Mechanical Design Process, 4th edition, McGraw Hill International, London.

[12] Varady T, Martin R R. Cox J, 1997, Reverse engineering of geometric models-an introduction. Computer-Aided Design 29(4):255-268.

[13] Botsch M, Kobbelt L, Pauly M, Alliez P, Lévy B, 2010, Polygon mesh processing CRC press.

[14] Durupt A, Remy S, Ducellier G, Eynard B, 2008, From a 3D point cloud to an engineering CAD model: a knowledge-product-based approach for reverse engineering. Virtual and Physical Prototyping 3(2):51-59.

[15] Ouamer-Ali M I, Laroche F, Bernard A, Remy S, 2014, Toward a methodological knowledge based approach for partial automation of reverse engineering. Procedia CIRP 21:270-275.

[16] Dryden I L, Mardia K V, 1998, Statistical Shape Analysis, John Wiley \& Sons, London.

[17] Kendall D G, 1984, Shape Manifolds, Procrustean Metrics, and Complex Projective Spaces, Bulletin of the London Mathematical Society 16:81-121.
[18] Anwer N, Schleich B, Mathieu L, Wartzack S, 2014, From solid modelling to skin model shapes: Shifting paradigms in computer-aided tolerancing, CIRP Annals Manufacturing Technology 63(1):137-140.

[19] Shapiro V, Solid Modeling, 2002, Handbook of Computer Aided Geometric Design, Elsevier, North-Holland, Amsterdam 473-518.

[20] Biasotti S, De Floriani L, Falcidieno B, Frosini P, Giorgi D, Landi C, Papaleo L, Spagnuolo M, 2008, Describing shapes by geometrical-topological properties of real functions, ACM Computing Surveys (CSUR) 40(4) 12

[21] Zhang D, Lu G, 2004, Review of Shape Representation and Description Techniques, Pattern Recognition 37(1):1-19.

[22] Koenderink J J, Van Doorn R J, 1992, Surface shape and curvature scales, Journal of Imaging and Vision Computing 10 (8):557-565.

[23] Zhao H, Anwer N, Bourdet P, 2013, Curvature-based Registration and Segmentation for Multisensor Coordinate Metrology, Procedia CIRP 10:112118.

[24] Xu S, Anwer N, Mehdi-Souzani N, 2015, Machining Feature Recognition from InProcess Model of NC Simulation, Computer-Aided Design and Applications 12(4):383-392 